\title{
Interference effect in the optomechanical stochastic resonance
}

\author{
Min Xie, Bixuan Fan, Xiaoli He, and Qingqing Chen \\ College of Physics and Communication Electronics, Jiangxi Normal University, Nanchang, 330022, China
}

\begin{abstract}
In this paper, we study the stochastic resonance (SR) effect in an optomechanical system driven by a strong coupling field and two weak signals in both semiclassical and quantum frameworks. In the semiclassical description, the SR phenomena are found at the cooperation of input signals and system noises. When two signals co-act on our system, the interference effect between the optically induced SR and the mechanically induced SR can be generated. In particular, a unique beating effect, which makes the SR effect robust against the initial phase difference of two signals, appears in the SR synchronization process with unsynchronized signals. In addition, the quantum stochastic resonance effect is numerically observed in the full quantum framework induced by pure quantum fluctuations.
\end{abstract}

\section{INTRODUCTION}

In recent decades, stochastic resonance (SR) has attracted considerable attention in various subjects [1, 2], such as physics, chemistry, biology and engineering science, for its intriguing and counterintuitive behavior in nonlinear dynamical systems, whereby a subthreshold input signal can be enhanced and optimized at an optimal noise level. SR was first proposed in 1981 to explain the periodicity of the ice ages [3, 4], and since then it has been demonstrated theoretically and experimentally in a variety of systems [1, 5], and applied to weak signal amplification [6, 7] and detection [8, 9]. A model most intensively investigated of SR is a bistable system subject to a feeble periodic signal and noise [10, 11]. It has been reported that SR can also occur in monostable systems [12-14] and multistable systems [15-17] as a consequence of nonlinearities in those systems. In addition, the SR phenomenon has also been extended into the quantum domain [18] and attracted increasing attention, such as the spinboson system [19], the micromaser system [20], the dissipative anharmonic oscillator [21], the quantum many-body system [22], the Dicke model [23] and the Jaynes-Cummings model [24].

It is known that nonlinearity is a key ingredient to induce the SR effect. With the development of fabricating optomechanical devices, the radiation pressure mediated optomechanical nonlinear coupling allows various nonlinear effects, such as bistability [25], multistability [17, 26, 27], instability [28, 29], and chaos [30, 31]. A standard optomechanical system (OMS) consists of an optical cavity where one of the end-mirrors oscillates and the radiation pressure on the moving mirror creates a nonlinear interaction between the optical mode and the mechanical mode. The study of SR in such a basic OMS may have great importance in understanding the nature of the SR effect [11, 32] and application in weak-signal detection [33].

In this paper we investigate the SR effect in an OMS subject to two weak signals (optical and mechanical signals) in the semiclassical framework and one weak mechanical signal in the quantum domain. In the semiclassical regime, the SR effect activated by the white noise is studied in three situations: a single signal, two synchronized signals, and two unsynchronized signals. The results show that the system modulated by a single subthreshold signal and a suitable noise can real- ize periodic interwell hopping synchronized with the signal, which is the typical SR effect. Interestingly, except for the conventional SR resonance peak in the signal-to-noise ratio (SNR) curve, a stage of decrease appears for lower signal amplitudes at a lower noise range due to the intrawell oscillation in a single well.

For the case of two signals, the system response can be interpreted as the interference between the two signals induced SRs. The interference of SRs usually occurs in the multistable systems [15, 17]. Here we present the interference of SRs occurring in a bistable system, which is jointly induced by an optical channel and a mechanical channel. We show that the constructive interference of two synchronized signals can reduce signal amplitudes for inducing SR, and the beating-like effect can appear when one signal is slightly detuned from the other. We find that the SR effect is robust and insensitive to the initial phase difference of signals as a result of beating.

In the quantum description, we explore the system stochastic dynamics induced by a weak mechanical force and quantum fluctuations using the quantum trajectory theory [34]. The results show that at zero temperature the quantum stochastic resonance (QSR) can also be observed in our bistable OMS and the system responses are synchronized to the external signal under appropriate system parameters at the optimal signal frequency.

This paper is organized as follows. In Sec. II, we introduce our model, and analyze the steady-state solutions as well as the stability. In Sec. III, we show the SR effects induced by a single modulated signal and thermal noise in the semiclassical framework, including the input-output synchronization and the resonance peak in SNR curve. Then, the combined effect of two signals in the SR process is discussed. In Sec. IV, we investigate the QSR effects subject to a weak mechanical force and pure quantum noise at zero temperature, and the residence time distribution and the system synchronous responses to the signal are described. In Sec.V, our conclusions are presented.

\section{MODEL}

We consider a standard OMS whereby the position of a mechanical oscillator modulates the resonance frequency of an optical cavity, as shown in Fig. 1 A mechanical mode with 
resonance frequency $\omega_{m}$ and an optical mode with frequency $\omega_{a}$ are coupled through the radiation pressure. The optical cavity is driven by a strong control field $E_{c}$ with frequency $\omega_{c}$ and a weak signal field $E_{s}$ with frequency $\omega_{s}$; a weak force $F_{s}$ with frequency $\omega_{f}$ acts on the mechanical oscillator. In a rotating frame, the Hamiltonian of the system reads $(\hbar=1)$

$$
\begin{aligned}
\hat{H}= & \frac{1}{2} \omega_{m}\left(\hat{x}^{2}+\hat{p}^{2}\right)+\Delta \hat{a}^{\dagger} \hat{a}+g \hat{a}^{\dagger} \hat{a} \hat{x} \\
& +E_{c}\left(\hat{a}^{\dagger}+\hat{a}\right)+E_{s}\left(e^{-i \delta t} \hat{a}^{\dagger}+e^{i \delta t} \hat{a}\right) \\
& +F_{s} \cos \left(\omega_{f} t+\phi\right) \hat{x},
\end{aligned}
$$

where $\hat{x}$ and $\hat{p}$ are the dimensionless position and momentum operators of the mechanical mode; $\hat{a}^{\dagger}(\hat{a})$ are the creation (annihilation) operators of the optical mode; $g$ is the optomechanical coupling; $\Delta=\omega_{a}-\omega_{c}$ is the detuning between the optical mode and the strong coupling field; $\delta=\omega_{s}-\omega_{c}$ is the frequency difference of the two external driving fields $E_{s}$ and $E_{c}$; whereas $\phi$ is the initial phase difference of the two weak signals $F_{s}$ and $E_{s}$.

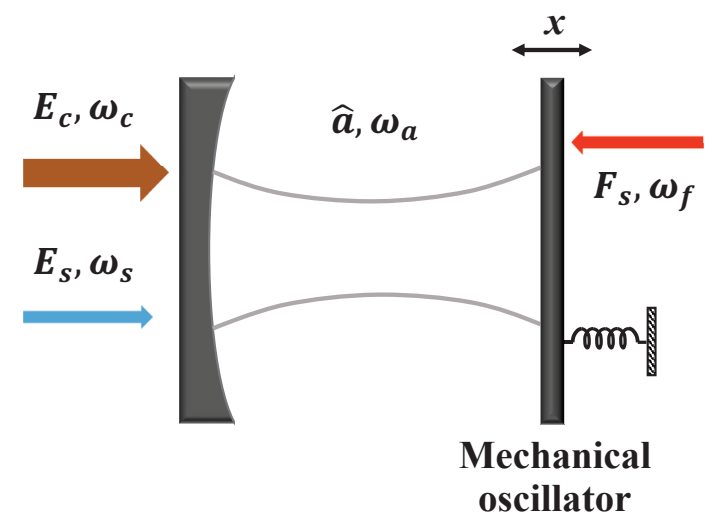

FIG. 1. Sketch of an optomechanical system. An optical resonator, driven by a strong control field $E_{c}$ and a weak signal field $E_{s}$, is coupled to the mechanical oscillator by radiation pressure. A weak force $F_{s}$ acts on the mechanical oscillator.

First, this paper deals with the system in the semiclassical description, thus we neglect quantum fluctuations of optics and mechanics. By using the Heisenberg equation of motion and phenomenologically adding thermal noise and damping terms, we can obtain the mean value equations of motion for classical system variables $\alpha=\langle\hat{a}\rangle, x=\langle\hat{x}\rangle$ and $p=\langle\hat{p}\rangle$ :

$$
\begin{aligned}
\dot{\alpha} & =-(i \Delta+\kappa) \alpha-i g x \alpha-i E_{c}-i E_{s} e^{-i \delta t}, \\
\dot{\alpha}^{*} & =(i \Delta-\kappa) \alpha^{*}+i g x \alpha^{*}+i E_{c}+i E_{s} e^{i \delta t}, \\
\dot{p} & =-\gamma_{m} p-\omega_{m} x-g|\alpha|^{2}-F_{s} \cos \left(\omega_{f} t+\phi\right)+\xi_{m}(t), \\
\dot{x} & =\omega_{m} p,
\end{aligned}
$$

where $2 \gamma_{m}$ is the mechanical damping rate, $2 \kappa$ is the optical decay rate and $\xi_{m}$ is the stochastic noise acting on the mechanical oscillator. For a high mechanical quality factor $Q=$ $\omega_{m} / \gamma_{m} \gg>1, \xi_{m}$ is the stochastic white noise and it obeys the $\delta$-correlation $\left\langle\xi_{m}(t) \xi_{m}\left(t^{\prime}\right)\right\rangle=2 D \delta\left(t-t^{\prime}\right)$ with the strength of noise $D \simeq \frac{\gamma_{m}}{2}(2 \bar{n}+1)$, where $\bar{n}=\left[\exp \left(\hbar \omega_{m} / k_{B} T\right)-1\right]^{-1}$ is the mean thermal excitation number[35, 36]. The thermal optical noise can be ignored at low temperatures as the thermal occupation of the optical mode is far below one.

By setting the time derivatives in Eqs. (2)-(5) to zeros, we can obtain the steady-state equation for mechanical position $x_{s}$,

$$
g^{2} x_{s}^{3}+2 g \Delta x_{s}^{2}+\left(\Delta^{2}+\kappa^{2}\right) x_{s}+\frac{g \omega_{m} E_{c}^{2}}{\left(\omega_{m}^{2}+\gamma_{m}^{2}\right)}=0,
$$

which is a cubic equation of $x_{s}$. As a consequence, three solutions of $x_{s}$ may exist in a certain range of system parameters, providing the possibility for bistability.

Following the linear stability analysis, we can rewrite the system operators as a sum of their steady-state values and zero-mean fluctuations, i.e., $\hat{y} \rightarrow y_{s}+\hat{y}$, and obtain the linearized equations of motion by ignoring high-order terms of fluctuations:

$$
\dot{y}=J \hat{y}+\xi
$$

$\begin{array}{llll}\text { where } \hat{y}= & {\left[\hat{a}, \hat{a}^{\dagger}, \hat{p}, \hat{x}\right]^{T}, \quad \xi} & = \\ {\left[-i E_{s} e^{-i \delta t}, i E_{s} e^{i \delta t},-F_{s} \cos \left(\omega_{f} t+\phi\right)+\xi_{m}(t), 0\right]^{T},} & \text { and }\end{array}$ the Jacobian matrix $J$ is given by

$$
J=\left[\begin{array}{cccc}
-i\left(\Delta+g x_{s}\right)-\kappa & 0 & 0 & -i g \alpha_{s} \\
0 & i\left(\Delta+g x_{s}\right)-\kappa & 0 & i g \alpha_{s}^{*} \\
-g \alpha_{s}^{*} & -g \alpha_{s} & -\gamma_{m} & -\omega_{m} \\
0 & 0 & \omega_{m} & 0
\end{array}\right] .
$$

The criterion of a stable solution is that the real parts of all eigenvalues of the Jacobian matrix $J$ are negative.

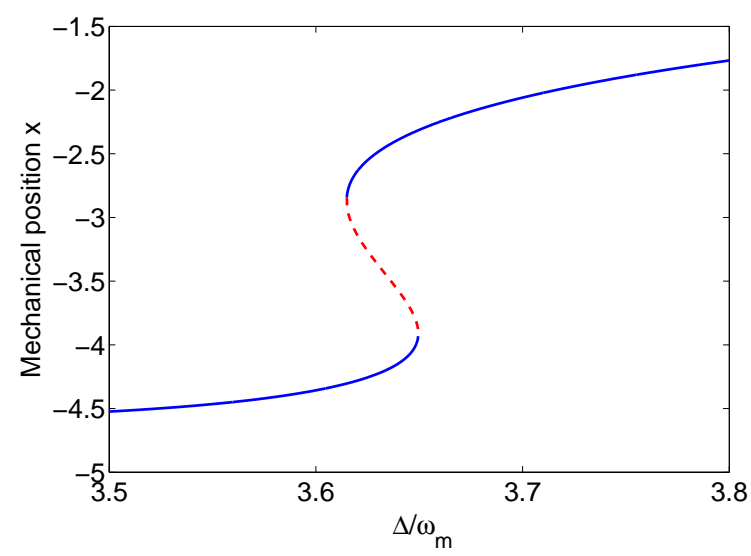

FIG. 2. The system stability diagram. Blue stands for the stable branches, and red stands for the unstable branch. The parameters are $\kappa=2.0 \omega_{m}, \gamma_{m}=2 \times 10^{-4} \omega_{m}, E_{c}=5.05 \omega_{m}$ and $g=0.72 \omega_{m}$.

The mechanical position $x$ versus the detuning $\Delta$ is illustrated in Fig. 2. It is clear that the system exhibits mechanical bistability and the mechanical position undergoes a transition from a single solution to three solutions. In the three-solution 
region, the upper and lower branches correspond to two stable solutions and the middle branch is unstable. In the following, we are interested in system dynamics in this bistable region.

To investigate the dynamics of the mechanical mode, we approximately derive the equation of motion merely for the mechanical mode under $\kappa>>\gamma_{m}, g$, where the dynamics of the optical mode is much faster than that of the mechanical mode and $\dot{\alpha}\left(\dot{\alpha}^{*}\right)$ can be set to zero to solve the stable value of $\alpha\left(\alpha^{*}\right)$. In this case, the equation of motion for the mechanical mode can be simplified to

$$
\begin{aligned}
\ddot{x}+\gamma_{m} \dot{x}= & -\omega_{m}^{2} x-\omega_{m}\left[g \frac{\left|E_{c}+E_{s} e^{-i \delta t}\right|^{2}}{(\Delta+g x)^{2}+\kappa^{2}}\right. \\
& \left.+F_{s} \cos \left(\omega_{f} t+\phi\right)-\xi_{m}(t)\right] .
\end{aligned}
$$

From this, we can obtain the effective potential function for the position of the mechanical oscillator in the absence of noise,

$$
\begin{aligned}
U(x)= & \frac{1}{2} \omega_{m}^{2} x^{2}+\omega_{m} F_{s} \cos \left(\omega_{f} t+\phi\right) x \\
& +\frac{\omega_{m}\left|E_{c}+E_{s} e^{-i \delta t}\right|^{2}}{\kappa} \arctan \frac{\Delta+g x}{\kappa} .
\end{aligned}
$$

As shown in Fig. 3, the effective potential varies periodically in the presence of the optical signal $E_{s}=0.021 \omega_{m}$ (left panel) or the mechanical force $F_{s}=0.032 \omega_{m}$ (right panel). Assuming that the two signals are synchronous over time, i.e., $\phi=0$, we can see that both signals give periodic modulation on the potential function and the modulations from the two signals are synchronized.
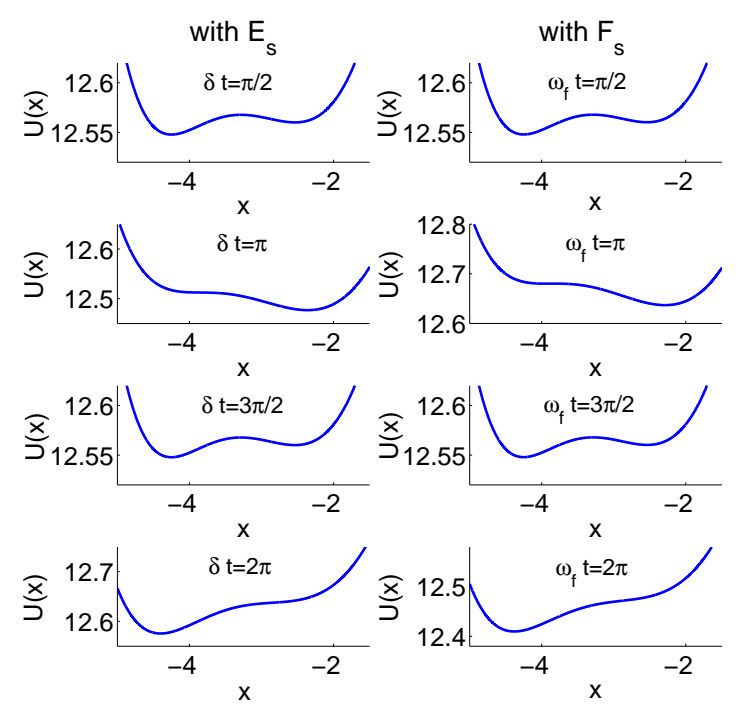

FIG. 3. Effective potential function of the mechanical position in one period with the optical signal $E_{s}=0.021 \omega_{m}$ (left panel) or the mechanical force $F_{s}=0.032 \omega_{m}$ (right panel). The detuning $\Delta=3.626 \omega_{m}$, the frequency difference $\delta=0.0006 \times 2 \pi \omega_{m}$ and the mechanical signal frequency $\omega_{f}=0.0006 \times 2 \pi \omega_{m}$, the initial phase difference $\phi=0$. The other parameters are the same as those in Fig. 2
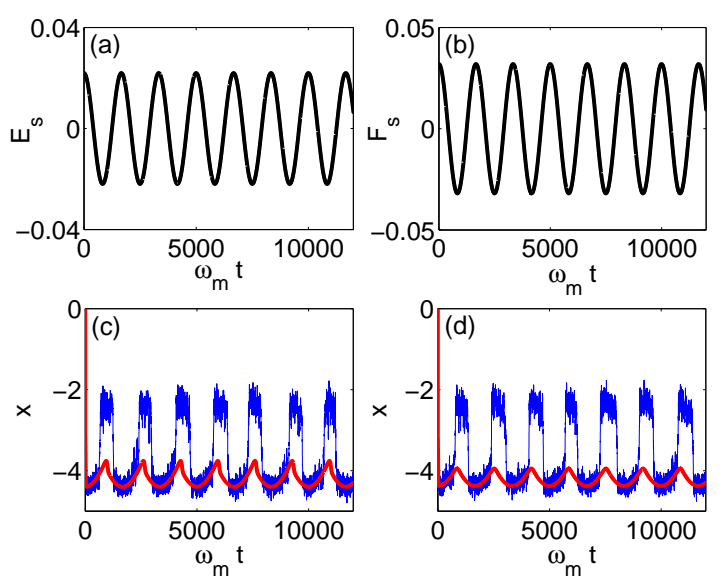

FIG. 4. Stochastic resonance with single modulation signal $E_{s}$ (left panel) or $F_{s}$ (right panel) in the semiclassical description. (a) and (b) are time evolutions of the signals; (c) and (d) present the steady-state position of the mechanical mode without mechanical thermal noise $D=0$ (red solid curves) and with $D=0.003 \omega_{m}$ (blue curves). The other parameters are the same as those in Fig. 3

\section{STOCHASTIC RESONANCE IN THE SEMICLASSICAL FRAMEWORK}

In this section, we present our main results in the semiclassical description: SR phenomena of our system under different thermal noise and driving signals. In all simulations, we assume that the mechanical oscillator is initially located at the original coordinate, i.e., $x(t=0)=0$. To observe noiseinduced system responses, the signals are chosen to be below thresholds. That means the mechanical oscillator can not cross the potential barriers only driven by the signals.

Figure 4 presents the system dynamics due to a single modulation signal $E_{s}$ (left panel) and $F_{s}$ (right panel), respectively, for the mechanical position $x$. It is clear that, in the absence of noise, the signals are too weak to drive the mechanical oscillator from one potential well into the other, and they can only drive small-amplitude oscillations within a single well as shown in the red curves in Figs. 4(c) and 4(d). By adding a certain amount of thermal noise $D=0.003 \omega_{m}$ to the system, the noise-assisted hopping between the double potential wells can be observed, and the hopping is exactly synchronized with signal frequencies. This is a typical signature of the SR effect.

Except for the input-output synchronization, a resonance peak in the relation of the SNR versus noise is another signature of SR. We now analyze this feature with the mechanical signal $F_{S}$ only under different intensities. Here we adopt the standard definition of the SNR: the signal in the power spectrum divided by the noise background at the driving signal frequency, i.e., $S N R=P_{s} / P_{n}$ [2].

In Fig. 5, the SNR of the mechanical signal versus the noise strength is plotted for three different amplitudes of the signal. For $F_{s}=0.032 \omega_{m}$, we can see a clear resonance peak in the SNR curve and the trend is quite standard. It is interesting to note that, for lower signal amplitudes $\left(F_{s}=0.015 \omega_{m}\right.$ and 


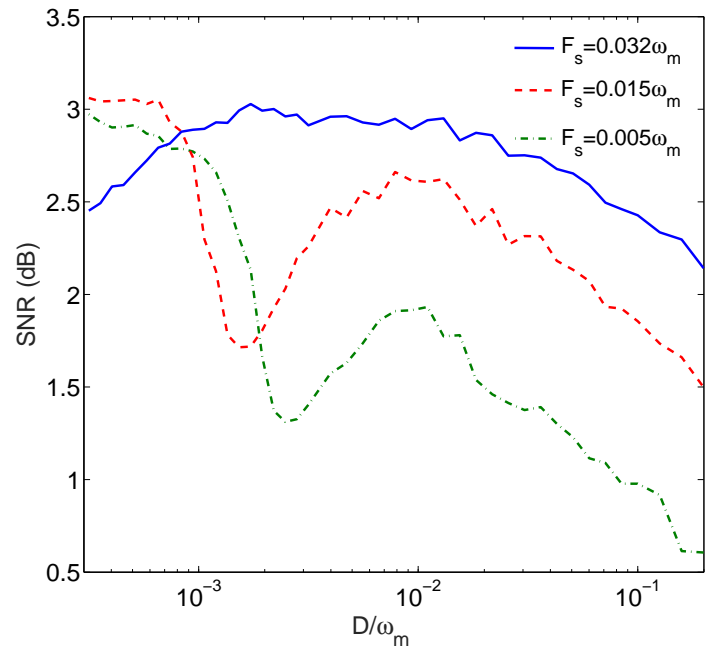

FIG. 5. The SNR in the decibel unit versus the noise intensity $D$ for different amplitudes of the mechanical signal $F_{s}$. The other parameters are the same as those in Fig. 4 except for $E_{s}=0$.

$\left.F_{s}=0.005 \omega_{m}\right)$, the SNR first experiences a stage of decrease other than the main SR resonance peak as the noise increases. In this decreased stage the mechanical oscillator is actually performing intrawell oscillation around the localized potential minima since the noise is too low to induce the interwell transition. As the noise intensity $D$ increases, the noise-assisted interwell hopping occurs, and the main interwell SR peak appears.

The system dynamics driven by a single mechanical or optical signal has been analyzed above. Now we turn our attention to the situation of two signals simultaneously acting on the system. Figure 6 shows the mechanical response of the system in the presence of two signals, and shows how their frequency difference affects the SR effect. First we consider the situation that two signals have the same modulation frequency and phases. With the matched modulation frequencies $\left(\delta=\omega_{f}\right)$ and appropriate noise, the periodic hopping between two stable states can be observed in Fig. 6a). The corresponding spectrum on the logarithmic scale is shown in Fig. 6(b), where a single peak is centered at the signal frequency of $0.0006 \times 2 \pi \omega_{m}$. It can be easily explained as the constructive interference caused by two synchronized signals. In addition, compared to the single signal case, the amplitudes of two signals required for SR to occur are substantially decreased, which is beneficial to the detection of weak signals in experiments. And the system parameters we have used are feasible for current experiment conditions [37].

When the modulation frequency difference $\Delta \omega=\delta-\omega_{f}$ is a small but nonzero value, some interesting phenomena take place, as shown in Figs. 6 (c) and 6(d). Here we choose $\Delta \omega=\delta / 10$. The interplay of the two signals results in a complicated beatinglike phenomenon where a slow modulation envelope and fast interwell and intrawell hoppings coexist. It is clear that the period of the slow envelope matches well with the curve $\cos (\Delta \omega t / 2)$, which is consistent with the theory of the beating signal. We can see that, in the regions with large modulation amplitude, the interference between the signal-induced responses is constructive and the system experiences periodic interwell transition. In contrast, for the regions with low modulation amplitude, their interference is destructive, and hence the mechanical oscillator can not cross the potential barrier and it oscillates inside a single well. Correspondingly, there are multiple peaks in the frequency domain for $\Delta \omega \neq 0$ [see Fig 6(d)]. Two main signal peaks at the input signal frequencies $\delta$ and $\omega_{f}$ and a difference frequency signal peak at $\delta / 10$ can be seen. Furthermore, the main resonance peak at $\delta$ in Fig 6 d) is lower than that in Fig. 6(b). It is not a surprising result since the interference of two signals is the strongest when they are exactly synchronized, as discussed in Ref. [17].
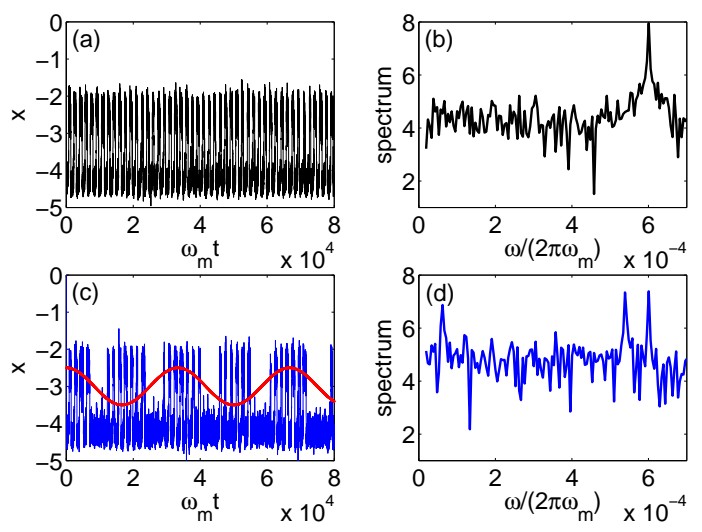

FIG. 6. The SR synchronization phenomena in the presence of two signals for (a) $\delta=\omega_{f}$ and (c) $\delta-\omega_{f}=\delta / 10$. (b) and (d) are the corresponding Fourier spectrums. The red solid curve in (c) is the function of $0.5 \cos (\Delta \omega t / 2)-3.0$. The other parameters are the same as those in Fig. 4 except $E_{s}=0.012 \omega_{m}$ and $F_{s}=0.015 \omega_{m}$.

Finally, we analyze the influence of the initial phase difference $\phi$ of the optical and mechanical signals on the SR phenomenon. From Fig 3, we know that when two signals are initially synchronized, i.e, $\phi=0$, their modulations on the potential function have the same pace and therefore they cause the best constructive interference in the SR phenomenon. For the other initial phases, their influences will be partly or fully canceled. To confirm this effect, we plot the mechanical responses for $\Delta \omega=0$ with $\phi=\pi / 2$ and $\phi=\pi$ in Figs. 7(a) and 7 (c). It is obvious that the SR effect diminishes to vanish as $\phi$ varies from 0 to $\pi$. However, the situation is dramatically different when the frequency difference of two signals is nonzero, i.e., $\Delta \omega=\delta / 10$. As shown in Figs. 7(b) and 7(d), the beating-like phenomenon always exists for different initial phases, and the input-output synchronization remains as good as that in Fig. 6(c). Therefore, the beatinglike phenomenon can make the synchronization behavior and the SR effect more robust to the initial phase fluctuations. 

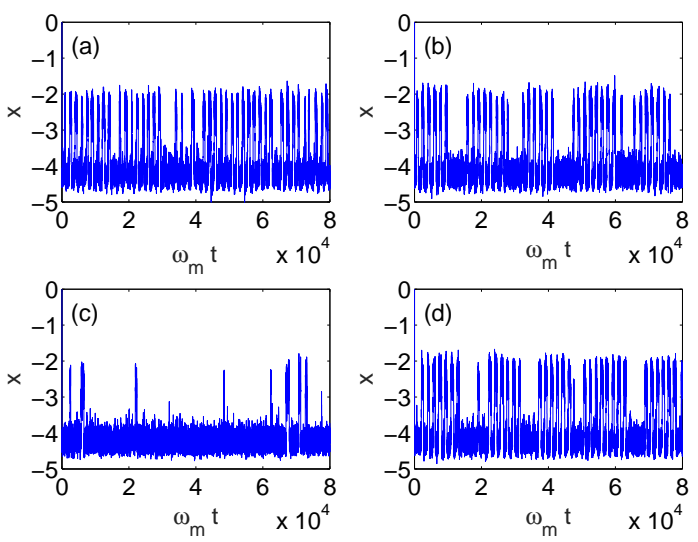

FIG. 7. The mechanical responses for several values of initial phase difference $\phi$ without (left panel) or with (right panel) frequency difference. (a) $\phi=\pi / 2, \Delta \omega=0$; (b) $\phi=\pi / 2, \Delta \omega=\delta / 10$; (c) $\phi=\pi, \Delta \omega=0$; (d) $\phi=\pi, \Delta \omega=\delta / 10$. The other parameters are the same as those in Fig. 6

\section{QUANTUM STOCHASTIC RESONANCE IN THE FULL QUANTUM FRAMEWORK}

In the preceding sections, we have studied the SR phenomena in the semiclassical framework. Now we turn to investigate the QSR effect induced by pure quantum fluctuations using the quantum trajectory method [34] at zero temperature. For a single trajectory, the system dynamic conditioned on noisy homodyne detection can be described by the stochastic master equation $(\hbar=1)$,

$$
\begin{aligned}
d \rho(t)= & d t\left\{i[\rho(t), \hat{H}]+\mathcal{D}[\sqrt{2 \kappa} \hat{a}] \rho(t)+\mathcal{D}\left[\sqrt{2 \gamma_{m}} \hat{b}\right] \rho(t)\right\} \\
& +d W(t) \mathcal{H}[\sqrt{2 \kappa} \hat{a}] \rho(t),
\end{aligned}
$$

where $\rho(t)$ is the density operator, $\hat{H}$ is the Hamiltonian of the OMS given in Eq.(11) and $d W$ is the Wiener increment, satisfying $\langle d W\rangle=0$ and $\left\langle(d W)^{2}\right\rangle=d t$. The superoperators $\mathcal{D}$ and $\mathcal{H}$ are defined as

$$
\begin{aligned}
\mathcal{D}[\hat{A}] \rho & =\frac{1}{2}\left(2 \hat{A} \rho \hat{A}^{\dagger}-\hat{A}^{\dagger} \hat{A} \rho-\rho \hat{A}^{\dagger} \hat{A}\right), \\
\mathcal{H}[\hat{A}] \rho & =\hat{A} \rho+\rho \hat{A}^{\dagger}-\operatorname{Tr}\left[\hat{A} \rho+\rho \hat{A}^{\dagger}\right] \rho .
\end{aligned}
$$

The corresponding homodyne detection current is given by

$$
I(t)=\sqrt{2 \kappa}\left\langle\hat{a}+\hat{a}^{\dagger}\right\rangle+d W(t) / d t .
$$

For convenience, we only investigate the QSR effect induced by a subthreshold weak force $F_{s}$ and the quantum noise. In Figs. 8 (a)-(c), we present the residence time distributions subject to the periodic weak force $F_{s} \cos \left(\omega_{f} t\right)$ for three different modulation frequencies $f=6 f_{0}, f_{0}, f_{0} / 6$, where $\omega_{f}=f \times 2 \pi \omega_{m}$ and $f_{0}=0.03$. The results show that the resonance can be achieved under appropriate parameters by varying the modulation frequency, distinguished by a separate peak of the distribution [see Fig. 8(b)].
In Figs. 8 (d)-(i), we show a few representative trajectories of the photon number $\left(\left\langle\hat{a}^{\dagger} \hat{a}\right\rangle\right)$ and the mechanical position $(\langle x\rangle)$. The system responses are synchronized to the signal best at the optimal modulation frequency $f_{0}$; a higher frequency $6 f_{0}$ or a lower frequency $f_{0} / 6$ leads to the destruction of synchronization, similar to the responses of the SR effect activated by thermal noise. However, due to different noise levels and system parameters, it requires a different time scale or frequency scale of the signal to satisfy the QSR matching condition.
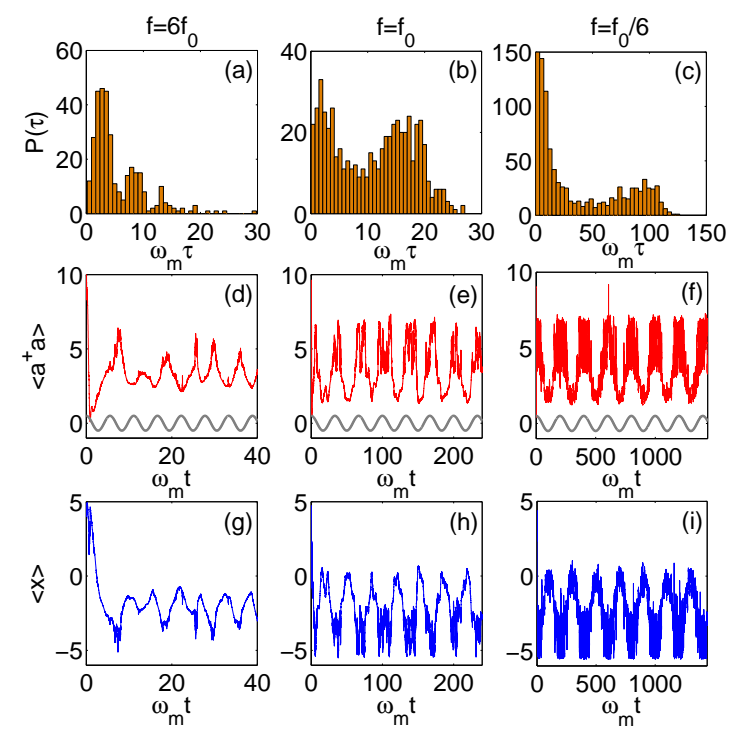

FIG. 8. The system responses subjected to a weak force and the quantum noise for three different modulation frequencies. (a-c) Histograms for the residence time distributions; (d-f) the average photon numbers. The gray curve is the function of $0.5 \cos \left(\omega_{f} t\right)$, characterized the period of the weak force; (g-i) the mechanical positions. The parameters are $E_{c}=5.15 \omega_{m}, E_{s}=0, F_{s}=\omega_{m}, \gamma_{m}=0.3 \omega_{m}$ and $f_{0}=0.03$. The other parameters are the same as those in Fig. 4

\section{CONCLUSION}

To summarize, we have investigated noise induced synchronization to external signals in a bistable optomechanical system in the semiclassical and quantum frameworks. Either a single optical signal or a single mechanical signal can induce the SR effect in our system. When the two external signals act on the system jointly, we can observe an interference of SRs, which leads to the beatinglike phenomenon depending on the frequency difference between signals. In addition, due to the beating-like effect, the input-output synchronization is more robust against the initial phase difference of two signals. Our results reveal that the optical pathway can be utilized to control the mechanical SR effect and detect the weak mechanical signal in a basic optomechanical system. Besides, we have numerically demonstrated the QSR effect induced by a weak force and pure quantum noise using the quantum trajectory 
method. The QSR effect, similar to the SR effect induced by the white noise, can be obtained under different system parameters at the optimal modulation frequency.

\section{ACKNOWLEDGMENTS}

The authors would like to thank Dr. Z. Duan for valuable suggestions. We gratefully acknowledge financial support from the National Natural Science Foundation of China under Grants No. 11504145 and No. 11664014 and from the Natural Science Foundation of Jiangxi Province under Grants No. 20161 BAB211013 and No. 20161BAB201023.
[1] L. Gammaitoni, P. Hänggi, P. Jung, and F. Marchesoni, Rev. Mod. Phys. 70, 223 (1998).

[2] T. Wellens, V. Shatokhin, and A. Buchleitner, Reports on Progress in Physics 67, 45 (2004).

[3] R. Benzi, A. Sutera, and A. Vulpiani, J. Phys. A 14, L453 (1981).

[4] R. Benzi, G. Parisi, A. Sutera, and A. Vulpiani, Tellus, 34, 10 (1982).

[5] V. S. Anishchenko, A. B. Neiman, F. Moss, and L. SchimanskyGeier, Usp. Fiz. Nauk 169(1), 7 (1999).

[6] P. Jung and P. Hänggi, Phys. Rev. A 44, 8032 (1991).

[7] R. L. Badzey and P. Mohanty, Nature(London) 437, 995 (2005).

[8] B. Kosko and S. Mitaim, Phys. Rev. E 64, 051110 (2001).

[9] F. Duan, F. Chapeau-Blondeau, and D. Abbott, Phy. Rev. E 84, 051107 (2011).

[10] L. Gammaitoni, F. Marchesoni, E. Menichella-Saetta, and S. Santucci, Phys. Rev. Lett. 62, 349 (1989).

[11] F. Mueller, S. Heugel, and L. J. Wang, Phys. Rev. A 79, 031804(R) (2009).

[12] J. M. G. Vilar and J. M. Rubi, Phys. Rev. Lett. 77, 2863 (1996).

[13] A. N. Grigorenko, S. I. Nikitin, and G. V. Roschepkin, Phys. Rev. E 56, R4907(R) (1997).

[14] N. V. Agudov, A. V. Krichigin, D. Valenti, and B. Spagnolo, Phys. Rev. E 81, 051123 (2010).

[15] P. K. Ghosh, B. C. Bag, and D. S. Ray, Phys. Rev. E 75, 032101 (2007).

[16] S. Arathi and S. Rajasekar, Phys. Scr. 84, 065011 (2011).

[17] B. Fan and M. Xie, Phys. Rev. A 95, 023808 (2017).

[18] R. Löfstedt and S. N. Coppersmith, Phys. Rev Lett. 72, 1947 (1994).

[19] M. Grifoni and P. Hänggi, Phys. Rev. Lett. 76, 1611 (1996).

[20] A. Buchleitner and R. N. Mantegna, Phys. Rev. Lett. 80, 3932 (1998).
[21] H. H. Adamyan, S. B. Manvelyan, and G. Y. Kryuchkyan, Phys. Rev. A 63, 022102 (2001).

[22] S. F. Huelga and M. B. Plenio, Phys. Rev. Lett. 98, 170601 (2007).

[23] D. Witthaut, J. Phys. B: At., Mol. Opt. Phys. 45, 225501 (2012).

[24] Q. Qiu, S. Tao, C. Liu, S. Guan, M. Xie, and B. Fan, Phys. Rev. A 96, 063808 (2017).

[25] Z. Duan, B. Fan, T. M. Stace, G. J. Milburn, and C. A. Holmes, Phys. Rev. A 93, 023802 (2016).

[26] F. Marquardt, J. G. E. Harris, and S. M. Girvin, Phys. Rev. Lett. 96, 103901 (2006).

[27] C. Schulz. A. Alvermann, L. Bakemeier, and H. Fehske, Europhys. Lett. 113, 64002 (2016).

[28] V. B. Braginsky, S. E. Strigin, and S. P. Vytchanin, Phys. Lett. A 287, 331 (2001).

[29] T. J. Kippenberg, H. Rokhsari, T. Carmon, A. Scherer, and K. J. Vahala, Phys. Rev. Lett. 95, 033901 (2005).

[30] T. Carmon, M. C. Cross, and K. J. Vahala, Phys. Rev. Lett. 98, 167203 (2007).

[31] L. Bakemeier, A. Alvermann, and H. Fehske, Phys. Rev. Lett. 114, 013601 (2015).

[32] F. Monifi, J. Zhang, S. K. Ozdemir, B. Peng, Y. Liu, F. Bo, F. Nori, and L. Yang, Nat. Photonics 10, 399 (2016).

[33] S. Aldana, C. Bruder, and A. Nunnenkamp, Phys. Rev. A 90, 063810 (2014).

[34] H. M. Wiseman and G. J. Milburn, Quantum Measurement and Control (Cambridge University, Cambridge, U.K., 2010).

[35] R. Benguria and M. Kac, Phys. Rev. Lett. 46, 1 (1981).

[36] S. Barzanjeh, D. Vitali, P. Tombesi, and G. J. Milburn, Phys. Rev. A 84, 042342 (2011).

[37] S. Groblacher, K. Hammerer, M. R. Vanner, and M. Aspelmeyer, Nature(London) 460, 724 (2009). 